\title{
Mixed Reality System for Ergonomic Assessment of Driver's Seat
}

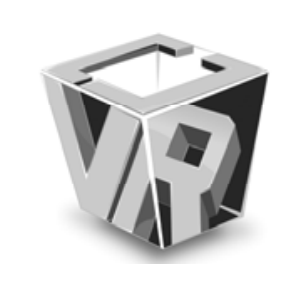

\author{
Giandomenico Caruso
}

Department of Mechanical Engineering - Politecnico di Milano 20156, Italy.

\begin{abstract}
Virtual Reality techniques are more and more used to validate the design hypotheses during different phases of the product development. In the automotive field, however, there are some types of analyses that are unlikely to be performed by using only a Virtual Prototype (VP) of the vehicle. Therefore, the trend is to develop systems -called seating bucks- that enable engineers to integrate virtual and real objects to provide a tangible interface for the VP of the vehicle.

This research aims at defining a new system and an evaluation protocol to improve, simplify and speed up the usual activities carried out for the ergonomic assessment of the driver's seat. In particular, we are interested in evaluating the ergonomics of car's dashboard with its knobs, buttons, display and other control systems. In this paper, we have described the development of this system by providing useful information about the physical issues, related to the seating buck structure, and about the development of the MR environment. The system and the evaluation protocol have been validated by performing several testing sessions with users. The tests confirm the effectiveness of our approach and have enabled us to achieve at interesting considerations.
\end{abstract}

Index Terms - Mixed Reality, Ergonomic analysis, Seating Buck, Haptic devices, Rapid Prototyping.

\section{INTRODUCTION}

In the last decades, automotive industry has strongly reduced the time to market of their products in order to satisfy the demand of global market. In recent years, automotive designers are making an ever-increasing use of Virtual Reality (VR) techniques in order to validate their own design hypotheses during different phases of the product development. The use of these techniques allows reducing the production of physical prototypes that are very expensive and requires long production times. On the other hand, there are some types of analyses that are unlikely to be performed by using only a Virtual Prototype (VP) of the vehicle. Therefore the trend in automotive field is to develop systems that enable engineers to integrate virtual and real objects in order to provide a tangible interface for the VP of the vehicle. An improvement of the user's interaction with the VP is needed when some aspects, as ergonomics, visibility, reachability, have to be evaluated. The design of a driver's seat is centered not only on aesthetic values and safety requirements, but it is also strongly related to ergonomic aspects, which obviously require the study of the interaction between the user and the components.

Nowadays, the evaluation of the comfort of the driver's seat is mainly performed in two ways: creating a physical prototype of the vehicle or using a driver's seat simulator named seating buck. Obviously the first solution is very expensive and does not enable us to perform comparative tests among different configurations. The seating buck, instead, is a configurable structure that allows us to simulate different driving seat set-ups. This structure, integrated with Mixed Reality (MR) technologies, allows us to perform different evaluation tests enabling the final users to see and interact in a natural way with the Mixed Prototype (MP) of the vehicle.

The main goal of the proposed system is to create a correct correspondence between the physical components of a dashboard and its virtual representations. In order to do that, we have used our MR environment that enables the users to see and interact in a natural way with both the physical and the virtual components. The physical prototype of the components has been obtained by using a rapid prototyping technique: specifically, we have developed the climate control system of a vehicle. In addition, we have integrated some haptic devices that realistically simulate the behaviors of the knob, slider and buttons, which are parts of the air conditioning system. These devices are mounted on a robotic arm that positions and re-positions them in the space, so as to allow us to evaluate the component reachability and the most appropriate positioning on the dashboard. The seating buck system has been validated through some testing sessions with users. During these sessions it has been possible to evaluate some ergonomic aspects of the dashboard. The involved users could interact with the seating buck components and communicate their opinions about the different proposed layouts of the dashboard.

The paper starts with a discussion on the limits of similar systems and on the problems of performing ergonomic analyses in a VR environment. In the third section, the main steps followed for developing the system are described by highlighting the original solutions that have been adopted. The fourth section describes the test protocol and the ergonomic analysis that have been carried out. Finally, in the fifth section, the results of our tests are discussed for assessing the usability and the effectiveness of the system. 


\section{RELATED WORKS}

The ergonomic assessment of driver's seat is a valuable analysis carried out in different ways and contexts. In aviation, the ergonomic assessment for cockpit layout of civil aircrafts is very important, because awkward posture caused by illogical layout bring pilot discomfort, fatigue and misplay, which would violate aviation security and be harmful for pilots. A typical approach is the use of Digital Human (DH) that can reduce the time and cost for cockpit development, as described in [1] [2]. In particular, the design of aircraft cockpit interfaces is an important focus for aviation ergonomics also for improving the battle efficiency of the pilot [3]. Using DHs provides an efficient solution for preliminary ergonomic evaluation. However, the reliability of the results are not comparable with those obtained in a real context [4], since sometimes it is not simple to extend the data, collected with DHs software, to the real conditions. For this reason, the trend is to carry out these kinds of evaluations with users by using physical prototypes that simulates the real functioning of the cockpit [5].

In automotive, such prototypes are called seating bucks and they derive directly from the driving simulators. Woon-Sung et al. [6] describe the driving simulator as a VR tool that gives to a driver-on-board the impression that he is driving an actual vehicle by predicting vehicle motion caused by driver input and feeding back corresponding visual, motion, audio and proprioceptive cues to the driver.

The driving simulators normally consist of several subsystems that allow engineers to reproduce actual driving conditions in order to evaluate driver-vehicle interaction. These systems are very complex and are mainly used for driver's safety analysis, and for training and vehicle tuning. The aim of the seating buck, instead, is not to simulate the dynamical behavior of the vehicle during the driving, but mainly to reproduce the interior of the driver's cab.

Today, in fact, the main application area of the seating buck is related to ergonomics studies that are performed to reduce tiredness and stress during driving by evaluating comfort conditions such as habitability, accessibility, visibility and reachability of the dashboard. The automotive seating bucks, in fact, allow simulating several vehicles interior so as to study different adjustments of primary driver controls, as for example seat, steering wheel, pedals, gearshift, etc.

Many research groups and industrial research centers use these kinds of systems to simulate the car interior and to perform visibility and ergonomic analysis of the driving seat.

Elasis research group, for example, developed a system based on a parametric driver's seat simulator, which supports automatic configuration of steering wheel, driver seat and pedals, coupled with an immersive VR environment [7]. The system allows evaluating and quantifying the comfort and the pleasantness of the car interiors by performing some tests with end users.

H. Salzmann et al. developed a two-user virtual seating buck system [8], which allows two users to take the function of the driver and co-driver respectively. Each user wears a tracked
HMD (Head Mounted Display) to see the virtual car interior from the respective point of view enabling them to correctly interact with the interface elements of a vehicle. In addition, this system gives the possibility of setting up three physical objects manually in the scene by using some flexi-holder.

These seating bucks are developed by using VR immersive environments that reduce the perceived realism of the scene: in fact, several users, especially when using a HMD, complain about an unnatural perception of space. In [9], Moehring et al. present an evaluation method for detecting possible causes of misperception focused on automotive industry scenarios by trying to mitigate perception errors by means of a depth of field blur applied to the virtual images. The misperception affects also the user's interaction that is fundamental during the ergonomic evaluations.

However, most of these systems provide only the possibility to set the configuration of the driver's seat, pedals and steering wheel while it would be interesting to simulate also the dashboard with its knobs, buttons, display and other control systems. Besides, it would be very useful to provide the possibility of configuring in real time the dashboard in order to compare different layout solutions by performing several ergonomic tests.

Another limit of these seating buck systems is related to the visualization and interaction issues. Actually, when the user is immersed in a virtual reality environment, the perception of the real objects present in the scene is distorted. Obviously this issue has a negative impact when the seating buck system is used to perform ergonomic and usability evaluations.

For these reasons, the trend for seating buck systems is to integrate physical object in the virtual scene and to develop, de facto, a MR environment. Toshikazu et al. [10] describe a MR system developed for the evaluation of automobile interior design and they observed that the sense of distance to the surrounding virtual environment and the sense of scale are improved becoming more direct than when only the visual sense is available. The sense of touch, given by physical objects, results to be effective when the computer-generated imagery is accurately registered and superimposed. For this reason in [11] we have analyzed and proposed a tuning procedure for setting stereo parameters in order to address this issue for our MR environment. In a recent work [12], we have demonstrated how our MR environment has been useful for the development and evaluation of an innovative tractor control system.

Starting from these considerations and from the results of our previous studies, we have concluded that it would have been useful to develop a seating buck system that allows us to improve the effectiveness of the ergonomic evaluation tests by introducing the comparison among different layouts of driver's seat components.

\section{MIXED REALITY SEATING BUCK}

The major requirements related to the design phase of the seating buck were to develop a flexible system, which allows us to represent different types of vehicle. In particular, we focused 
on the development of a system able to optimize the design process of driver's seat layout. The system consists of a flexible structure accommodating the components (seat, steering wheel, pedals, etc.). In addition, the system has been integrated with a robotic arm that allows us to change dynamically the position and the orientation of a car dashboard mock-up, and also to move some car's control devices, in order to determine their best configuration (in terms of haptic feedback and position).

The seating buck system has been developed basing on Virtual Prototyping techniques: virtual humans have been used to validate its functionalities. Subsequently, this structure has been developed, mainly by using standard alloy profiles, and integrated with a MR environment in order to provide the final user with the possibility of interacting with the system in a natural way. The users can see and interact with virtual and physical components directly with their own hands.

\subsection{Specifications for seating buck structure development}

The first step, in the design of the seating buck, has been the definition of the configuration of the structural components and of the various elements required in order to simulate different types of driver's seats. In this first phase, which mainly consists in the data collection and the definition of specifications, we have considered only the primary elements of the vehicle.

The dimensions, which determine the interior space and access of a vehicle, are taken from the document Society of Automotive Engineers SAE J1100 [13]. The system has been defined on the basis of a coordinate system, whose origin is the Accelerator Heel Point (AHP) on which the foot pivots in order to press the accelerator pedal. In Fig. 1 a schematic representation of these dimensions are shown.

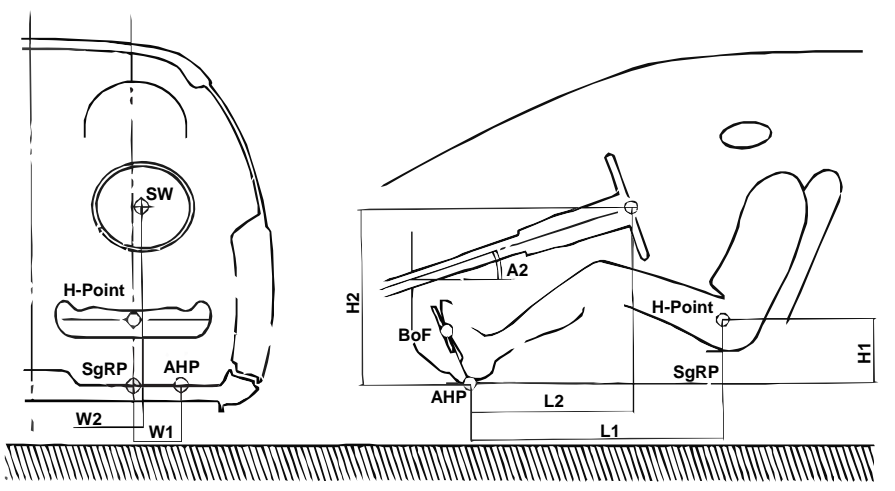

Fig. 1. Schematic representation of the dimensions that define the interior space and access of a vehicle [13].

The best pedals position is usually defined around the AHP and the Ball Of Foot (BOF), which is the point where the user puts the thrust of the foot on the pedal. An additional reference point, which has been considered, is the H-point that is the pivot of the torso and thigh. Starting from this point, the positions of the other components have been defined.

It is clear that these points are related to the user's sizes and thus they deeply influence the degree of postural comfort. Therefore it is important to take into account, since the beginning of the analysis, that the range of population to be considered may include people whose measures can be widely different.

TABLE 1: MEASUREMENTS USED TO DEFINE DRIVER'S SEAT.

\begin{tabular}{|l|c|l|c|}
\hline Component & Indicator & \multicolumn{1}{|c|}{ Measurement } & Code \\
\hline Pedals & AHP & Zero Axis & \\
\hline \multirow{3}{*}{ Seat } & \multirow{3}{*}{ H-Point } & Z distance & H1 \\
\cline { 3 - 4 } & & X distance & L1 \\
\cline { 3 - 4 } & \multirow{4}{*}{$\begin{array}{l}\text { Steering distance } \\
\text { Wheel }\end{array}$} & Z distance & W1 \\
\cline { 3 - 4 } & \multirow{2}{*}{ SW } & X distance & H2 \\
\cline { 3 - 4 } & & Y distance & L2 \\
\cline { 3 - 4 } & Angle respect X axis & A2 \\
\hline \multirow{3}{*}{ Gearshift } & \multirow{2}{*}{$\begin{array}{c}\text { Top of the } \\
\text { gearshift }\end{array}$} & Z distance & X distance \\
\cline { 3 - 4 } & & Y distance & L3 \\
\hline
\end{tabular}

Table 1 shows a simplified version of the characteristic measurements that allow the definition of the relative positions of each elements of the driver's seat in respect to a reference point. According to this scheme, the reference point of the system is represented by the AHP.

The translation and angular motion values for each element of the vehicle, suggested by the SAE standards, are identified in a measurement range obtained through a statistical analysis conducted on various vehicles [7]. Usually, automotive manufacturers refer to SAE standards that define the best dimensional relations. These standards provide guidance, which results to be too complex for the aim of this phase of preliminary configuration based on a survey of the various vehicles present on the market. To avoid the complexity of these regulations, we have planned to define the structural measures for different types of vehicles to use for detecting the actual excursions, which result to be necessary for configuring each seat element.

Therefore, we have chosen five different vehicles, as a small statistical sample, in order to define the necessary measurements. The five vehicles selected are the following: Volkswagen Golf, Lancia Y, Toyota Corolla, Mercedes C220, Alfa Romeo 166.

The methodology adopted for gathering all the measures has been described in [14], which is a document published yearly that collects the result of an even closer collaboration among car manufacturers. Since these statistical measurements are essential only to define a range of arbitrary mobility of the seating buck elements, it was not necessary an over accuracy. As a result of the measurements, the data collected have been useful to define the ranges of possible movements of each element of the structure, providing the seating buck with a sufficient flexibility to simulate a variety of different car models.

During the measurement phase we have decided to put as zero-system the AHP, since it is not subject to any regulation of position (as for the seat or steering wheel). However, since the seat is very cumbersome and moreover it is the heaviest element of the structure, we set just one Degree Of Freedom (DOF): the vertical translation. So, we defined as a zero-system the projection of the H-point on the footrest plane: the Seating Reference Point (SgRP). It was more convenient to use as a reference for the zero-system a point related to the seat, because 
this is the element with the lesser number of DOF.

TABLE 2: DEGREES OF FREEDOM OF SEATING BUCK ELEMENTS.

\begin{tabular}{|l|l|c|c|}
\hline Element & \multicolumn{1}{|c|}{ DOF } & Ref & $\begin{array}{c}\text { Bounds of } \\
\text { positioning }\end{array}$ \\
\hline Seat & vertical translation & $\mathrm{z}$ & $20 \div 45 \mathrm{~cm}$ \\
\hline \multirow{4}{*}{$\begin{array}{l}\text { Steering } \\
\text { Wheel }\end{array}$} & longitudinal translation & $\mathrm{x}$ & $25 \div 45 \mathrm{~cm}$ \\
\cline { 2 - 4 } & lateral translation & $\mathrm{y}$ & $-2,5 \div 2,5 \mathrm{~cm}$ \\
\cline { 2 - 4 } & vertical translation & $\mathrm{z}$ & $40 \div 85 \mathrm{~cm}$ \\
\cline { 2 - 4 } & rotation & $\mathrm{xz}$ & $-10 \div 45 \mathrm{deg}$ \\
\hline \multirow{4}{*}{$\begin{array}{l}\text { Pedals } \\
\text { apparatus }\end{array}$} & longitudinal translation & $\mathrm{x}$ & $70 \div 105 \mathrm{~cm}$ \\
\cline { 2 - 4 } & lateral translation & $\mathrm{y}$ & $5 \div 20 \mathrm{~cm}$ \\
\cline { 2 - 4 } & vertical translation & $\mathrm{z}$ & $5 \div 20 \mathrm{~cm}$ \\
\cline { 2 - 4 } & rotation & $\mathrm{xz}$ & $-10 \div 65 \mathrm{deg}$ \\
\hline \multirow{3}{*}{ Gearshift } & longitudinal translation & $\mathrm{x}$ & $15 \div 50 \mathrm{~cm}$ \\
\cline { 2 - 4 } & lateral translation & $\mathrm{y}$ & $25 \div 40 \mathrm{~cm}$ \\
\cline { 2 - 4 } & vertical translation & $\mathrm{z}$ & $30 \div 70 \mathrm{~cm}$ \\
\hline
\end{tabular}

Table 2 shows the bounds of positioning that we have elaborated for each element according to the DOFs. We have so far considered the four primary elements characteristic of each vehicle, which are the seat, the steering wheel, the pedals apparatus and the gearshift. Subsequently, we have decided to not replicate physically the gearshift since it is not an element investigated in our ergonomic evaluations. However, we have reported the data related to the gearshift, since they will be useful for further seating buck developments.

Once defined the bounds of positioning for each element, which makes up the structure, the degree of freedom of the seating buck has been assessed. Then, we have started the design phase. The whole design phase of the seating buck has been conducted by using DHs in order to design a proportioned structure and to validate our design choices (Fig. 2).

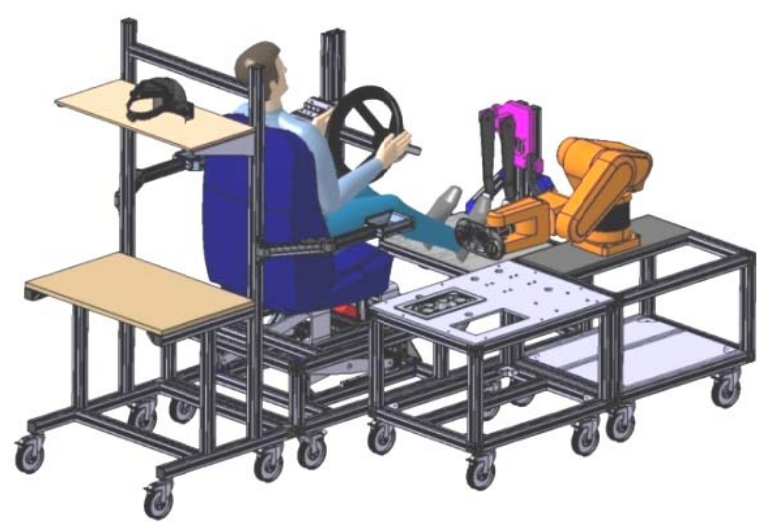

Fig. 2. The virtual representation of our seating buck.( Color Plate 9 )

The accessibility and the configurability of the system are two of the main aspects investigated through the DH simulation. To do that, we have elaborated some routines that enable us to simulate, with the $\mathrm{DH}$, the tasks that the real user has to carry out during the testing session. From these simulations, useful information emerged in relation to the performing of the testing session. In particular, such data have been used for defining the protocol of the pilot test that will be described in the next sections.
By using similar routines, we have also simulated the movements of the robot for defining the best path that the robot has to tread for configuring the system. Such simulation allowed us to avoid that the robot, during its movement, hits the structure or the user, thereby preventing any injuries.

\subsection{Structure of seating buck}

The major design requirements of the whole system concerned economy, transportability and upgradeability. Therefore, we have developed the seating buck structure by using Bosch Rexroth components [15] that provide a high level of flexibility and an easy rearrangement of the elements. The structure layout has been designed so as to have a basic frame. It consists of a main module that connects together all the other modules, which can be easily unhooked and transported, as they are equipped with wheels. Besides, Bosch Rexroth aluminium profiles make the structure being easily upgraded for future developments.

The seating buck is made up of four modules that have been named: Main module, Robotic arm module, Rack module, and VR equipments module.

The Main module is further divided into four parts: the skeleton of the structure, the pedals mechanism, the steering-wheels mechanism and the mechanism of the seat.

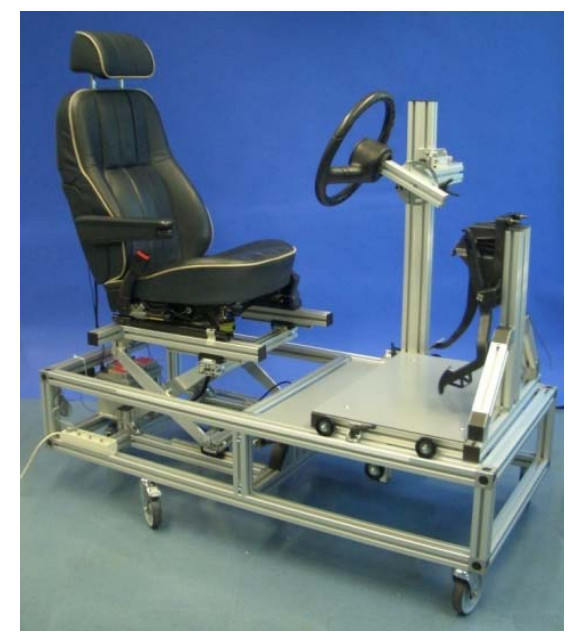

Fig. 3. The Main module of the seating buck. ( Color Plate 10 )

The skeleton is a simple frame where all the other modules and the principal elements have been hooked together (Fig. 3).

As said before, we have decided to configure each part of the seating buck starting from the seat position, which is provided only with the vertical translation. Therefore the pedals apparatus replaces the longitudinal translation of the seat, bringing near and moving away from the seat, and thus from the H-point, the whole footrest identified by the AHP point. The whole apparatus takes advantage of the particular shape of the profile of the skeleton as rails on which the footrest slides. In addition each pedal, which is mounted on the footrest frame, is provided with one DOF: the rotation in the $\mathrm{x}-\mathrm{z}$ plane. Thus, it is possible to set the angle of the pedals at any value in the above-described range. The steering wheel presents four DOF: three for translations and one for rotation in the $\mathrm{x}-\mathrm{z}$ plane. As 
regards the seat, we have developed an actuated mechanism, based on the Scott-Russel mechanism [16], in order to simply manage the vertical DOF of the seat.

The Robotic arm module is a simple independent structure that allows using the robotic arm in different configurations.

The Rack module allows us to support all the devices and tools that the robot needs to move during the execution of evaluation, and hence it is placed close and connected to the Robotic arm module. Behind the main structure, we have also placed an independent $V R$ equipment module as the storage space of the VR equipments. As already explained, these modules are physically independent from each other and this provides the possibility of a rearrangement of the modules considering of future developments as well as an easy transportability of the structure.

\subsection{Mixed Reality environment}

Our MR environment consists of different hardware and software components that we have integrated for enabling the user to correctly interact with the simulated driver's seat, as shown in Fig 4. The selection of the hardware, used for our MR environment, is due to our previous experiences [12].

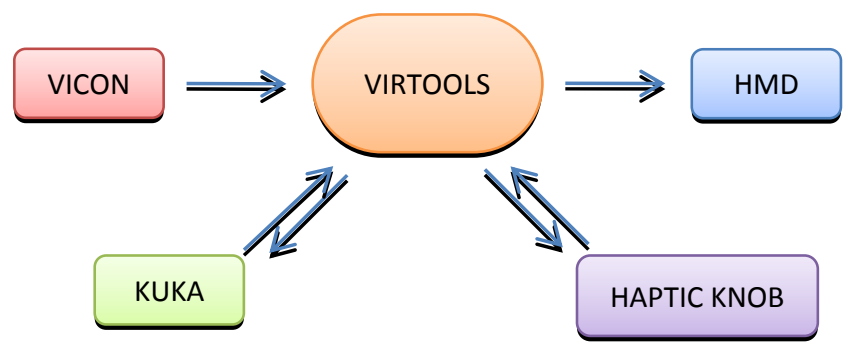

Fig. 4. The main hardware and software components of our MR environment.

The visualization of the interior of the vehicle was provided by using the Optical See-Through Head Mounted Display (OST-HMD) nVisor ST [17]. The use of such HMD allows the simultaneous visualization of both virtual and physical objects. The user will continue to see himself inside the scene; this condition increases his sense of presence within the environment. In order to improve the visualization of the arm of the user we have used a lighting system directed onto the subject. This contributes to increase the occlusion of the virtual objects with the real ones when it is necessary

The virtual representation of the driver's seat components must be registered with the real ones. For this reason, in order to obtain the user's head tracking and the registration of the seating buck in the virtual environment, it is necessary to use a tracking system. We have used the VICON 460 optical motion capture system [18] equipped with 6 infrared cameras.

The robotic arm, which we have used, is the industrial manipulator KR3 from Kuka Robotics [19]. It is a 6-axes robotic arm with a payload of $3 \mathrm{Kg}$, repeatability of $\pm 0,05 \mathrm{~mm}$ and a work envelope volume of $0,679 \mathrm{~m}^{3}$.

The end-effector, which we have used for a fast lock of our equipments, is an electromagnet (MAX. payload 40Kg) activated by the host computer. In the set-up of our tests, the robotic arm carries, alternatively, a haptic device and a dashboard prototype manufactured through a rapid prototyping technique (Fig. 5).

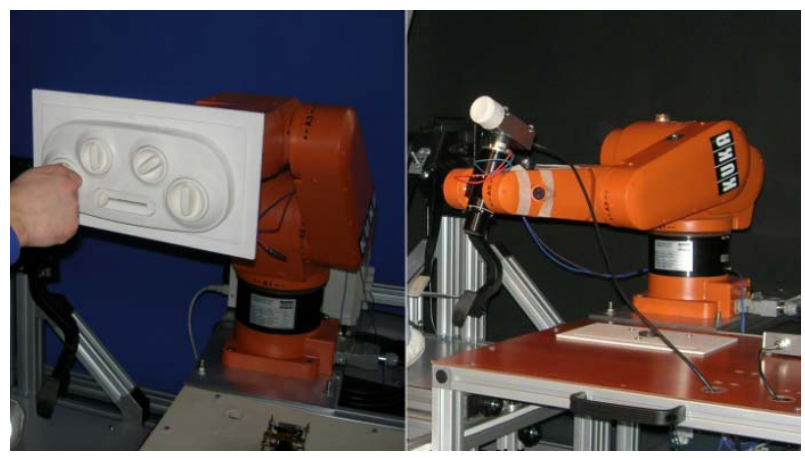

(a)

(b)

Fig. 5 Robotic arm with the dashboard prototype (a) and the haptic device (b).

The haptic device is the new version of the haptic knob developed by our research group [20]. It is a device with 1 Degree Of Freedom (DOF), which allows the simulation of a real mechanical knob. The knob is connected to a control unit that reads the information from the knob and generates the voltage required to obtain the proper haptic feedback. The haptic feedback is defined both in terms of step numbers, rotation interval and torque, which can be constant or variable, as desired.

The software used to develop our MR environment is the Virtools software [21]. This software is a development platform widely used in industrial fields for the development of interactive 3D applications. It is natively capable of integrating different third-parts hardware components by means of visual programming, named building blocks, and gives the possibility to add new ones. In particular, we have developed two new building blocks in order to connect our haptic knob to the KR3. The algorithm running on the Kuka KR3 has been developed by using its proprietary language, which provides the commands to move the mechanical arm by using common control-flow statements (e.g. IF, WHILE, DO-WHILE, LOOP).

\subsection{Software implementation}

The aim of our software implementation is to integrate all the above-described hardware so as to make automatic and safe the configuration of the MR environment during the testing sessions.

At first, we have imported in the Virtools software the VP of our seating buck in order to quickly register the VP of the driver's seat in the right position. The right correspondence between the virtual seating buck and the real one is constantly guaranteed by the tracking system. Unfortunately, the technology of the OST-HMD does not enable us to compare the real and the virtual objects seen by the user and it is not possible to evaluate the accuracy of the registration. Consequently, we have implemented a procedure that enables the users to manage the virtual images, by means of a remote control, for compensating any registration error. Thanks to the remote control the user can move the stereoscopic images in the three-dimensional space comfortably, thus obtaining the 
perfect overlap between virtual objects and real ones.

The MR environment assumes that the user's point of view is tracked according to the virtual objects, which have to be superimposed on the corresponding real ones. The tracking, as said before, is provided by the Vicon tracking system that is able to recognize and track different maker-sets, which we set on the OST-HMD, on the right user's wrist and on the steering wheel. All the tracking data refer to the origin of the Vicon, which can be positioned inside the control volume. For convenience, we set the origin on the back-right edge of the seating buck skeleton module. The coordinates of the different components, which are transferred to the Virtools by Ethernet connection, are used to manage position and orientation of the virtual camera, according to the user's point of view, and position and orientation of virtual steering wheel, according to the real one.

Subsequently, we needed to control and monitor the robot functioning through Virtools. For this reason, we have developed a new building block, written in $\mathrm{C}++$, thanks to which it is possible to retrieve information, from the controller of the KR3, about robot axes, position and orientation of the end-effector and, furthermore, it is possible to send commands for moving the KR3 in pre-registered positions or according to the user's preference. The building block communicates with the KR3 through an algorithm that we have implemented on the robot controller by using its language. Firstly, the software initializes the robot, then moves the KR3 to its HOME position and finally initializes, as a client, the Ethernet connection, with the application server developed into our Virtools building block. Now, the KR3 is waiting for external commands formatted into a specific XML schema. To stop remotely the robot during its movement, we have created a so-called INTERRUPT function that is able at any time to abort any of the activities of the KR3.

The origin of the KR3, obviously, is different from the origin of our MR environment, since the KR3 is mounted on a mobile separate module. Subsequently, we elaborated a calibration procedure in order to set the robot's root origin, according to the origin of the environment. The calibration procedure consists in acquiring the coordinates of a calibrated frame fixed on the center of the end-effector while it is moved by the KR3. The acquired data are subsequently elaborated to define the transformation matrix that converts the coordinates of the end-effector according to our system's origin. This procedure is needed only if the KR3 module is moved or when the tracking system needs to be re-calibrated.

Another building block has been developed for controlling the haptic knob for managing the devices directly by Virtools. This building block allows us to set the behavior of the device (torque, number of steps, end-stop) and for acquiring in real time the value of the knob angle in order to move the virtual representation of the knob according to the physical one.

\section{TESTING SESSION}

The main purpose of our work is to provide an effective system for performing several ergonomic tests of cars interior and to define a useful protocol for comparing different design hypotheses. In particular, our test aims at assessing the comfort of various layouts of the climate control system interface. From this point of view, our seating buck system could be very useful for conducting such kind of tests since, thanks to its quick configurability; it enables us to easily acquire the data relative to ergonomic tests with several final users. At the same time, we have used such tests for assessing the usability and reliability of the seating buck system.

The definition of a pilot protocol for conducting the analysis is always necessary and, for this reason, we have decided to define a comparative evaluation of two different layouts related to the knob position and to the behavior of the air-conditioning interface. Comparative ergonomic tests provide more effective results, as well as useful information, by reducing efforts and time to perform it [22].

The selection of the testing modalities can be heavily characterized by some decisions that have to be taken in advance. Some protocols, for example, suggest the selection of expert-users sample for conducting heuristic evaluations [23], or at least they should have a certain degree of freedom of expression for the tester and a certain degree of freedom both during the tasks and the environment exploration. The tester has to be able, in this case, to convey his feelings through "thinking aloud" techniques [24] or by compiling a final evaluation report. These procedures are particularly suitable for the detection of usability problems. The procedures can be thought as a sort of brainstorming concerning the current research problem, and enable the user to explore in the most natural way the proposed situation. On the other hand, these tests allow the emerging of all the problems and constraints or limits present in the system during its use. Heuristic evaluations are always carried out by inviting expert users, in order to shed light on any critical elements of the object under analysis and to provide guidelines useful for the setting of structured tests, basing the guidance of the procedure on fundamental aspects [25]. Actually, this kind of test is mainly used for assessing the usability of software applications while we have extended this investigation method to our seating buck system and, in particular, by evaluating the five qualitative aspects defining the usability, as described in [26]:

- Learnability: easiness of succeeding in the basic operations at first attempt;

- $\quad$ Efficiency: the pace in carrying tasks once that the user has understood the functioning of the system;

- $\quad$ Memorability: easiness experienced by users in re-obtaining the same level of confidence with the system after a period of non-use;

- $\quad$ Errors: quantity of errors, seriousness and recovery possibilities;

- $\quad$ Satisfaction: enjoying in using the system.

According to the selected objectives and to the modalities used for performing the analysis, we have studied four of the 
TABLE 3: EVALUATION SCALE ELABORATED STARTING FROM THE SAE STANDARDS.

\begin{tabular}{|c|c|c|c|c|c|c|c|c|c|c|}
\hline & \multicolumn{5}{|c|}{ Not acceptable } & \multicolumn{5}{|c|}{ Acceptable } \\
\hline Scale & 1 & 2 & 3 & 4 & 5 & 6 & 7 & 8 & 9 & 10 \\
\hline Meaning & poor & very bad & bad & fairly bad & $\begin{array}{c}\text { light } \\
\text { acceptable }\end{array}$ & acceptable & fairly good & good & very good & excellent \\
\hline Feeling & \multicolumn{3}{|c|}{ intolerability } & \multicolumn{2}{|c|}{ discomfort } & \multicolumn{2}{|c|}{ light discomfort } & \multicolumn{3}{|c|}{ comfort } \\
\hline
\end{tabular}

five qualitative components above described. We excluded the memorability aspect since this datum is not significant for the purpose of our tests.

\subsection{Preliminary questionnaire}

Before the test started, we asked the testers to read an instruction note and to fill in a brief questionnaire. The questionnaire is divided into two sections. The first one concerns the personal information of the user: age, gender, weight, height, and visual ability of the user. The second section aims at gathering information about the user's habits on car driving. It is important to know which model of car the user is familiar with, in order to understand any potential sense of extraneousness in the event that the configuration of the car used in the tests has a completely different asset. The evaluation of these data is not very useful for the purpose of the testing session since the sample involved is too small for statistical extrapolations. However, our aim has been defining a questionnaire that will lead us to better understand the reasons on which ground the users' answers. Such analysis could be applied to the comments provided, leading to a critical analysis of the results and to a further awareness for the selection of the tester samples in future tests.

\subsection{Evaluation scale}

A detailed and rigorous protocol has been initially defined so that the repeatability of the questions and the operations has been guaranteed allowing the subsequent and coherent comparison among the obtained data.

In order to achieve this purpose, the questions have to be posed in an unambiguous manner, and hence the most suitable way has been identified in the setting of multiple-choice questionnaires. Three types of questions have been submitted to the testers and each type of question presumes three different types of answers:

\section{- $\quad$ Affirmative/negative;}

- $\quad$ Multiple choices: from 3 to 5 possible choices;

- Qualitative evaluation (which is expressed in a numerical value from 1 to 10 ).

It is fundamental that the user clearly understands the proposed questions, and that he has both the appropriate knowledge and tools for a mindful answering. In particular, the third type of answer, which includes a qualitative evaluation, as well as emotional, represents the most critical point.

This kind of tests requires a specific connection between the evaluation expressed by the user and a certain type of feeling. Moreover, a detailed explanation of the meaning of each evaluation could be very useful for the comprehension and the verbalization of the perceptions. In this way, the expressed evaluation seems to be more reasoned and less instinctive, so that the user is encouraged to use all the levels present in the metrics.

In order to make univocal the interpretation of the metrics used to analyze the obtained results, it is advisable to precisely explain the metrics itself. Consequently, we used an evaluation scale, represented in Table 3 that we elaborated starting from the SAE standards [27]. This scale has been fixed to the steering wheel' structure positioned in front of the user sitting in the seating buck.

With the aim of providing two different situations to be compared, one of the knobs of the air conditioning system could be moved and placed in different positions within the driver's cab. In this way, we can also study the convenience of use, due to the size and placement of the knobs, as well as the reachability of the control devices that can be put in relation with the percentiles of the users and the ergonomic analysis. This possibility of the double-placement is easily performed by the robotic arm, which can interactively modify the position of the dashboard in the environment. Except the steering wheel, the rest of the dashboard can slightly change its position both on the horizontal and on the vertical plane. Since these changes are very small, they do not alter the testing results, and in the meanwhile they allow the studying of the controls placement.

It is evident that if the central purpose of the study were related to the analysis of the controls placement, it would have been necessary to radically change the whole structure of the dashboard on the basis of the interfaces placement. However, we did not focus on this issue, and hence we have conducted our tests aiming at validating the system functioning, at highlighting the limits, and at creating the basis for future developments.

The existence of two configurations determines inevitably a transitional moment, when the system needs to be reconfigured: in particular, the robotic arm has to replace the interface under analysis. The virtual image, which is projected in the OST-HMD, has to be changed for all the simulations that require it. The user does not have to spot directly these changes; otherwise he will previously understand the difference between the two proposed layouts.

A second important aspect, related to the evaluation modalities for the comparison between the two situations is the 
order in which the two options are showed. It is possible to acknowledge that the first configuration will be evaluated in a natural and spontaneous way, whilst the second configuration will be evaluated on the basis of a comparison with an unconscious attempt of making coherent the answers. This aspect has to be necessarily taken into account, during the analysis of the data. Therefore, we have decided to propose the different situations each time in a diverse order for two basic reasons: first to minimize the effect of this phenomenon and second to inquire on its entity. After having defined the operative modalities necessary to carry out the analysis, we have detailed the organization of the tests.

\subsection{Haptic behaviors configuration}

The great freedom offered by the programmable haptic device allows us to analyze the perception of physical behavior command in order to quickly evaluate the effectiveness of feedback among different behavioral alternatives. Two different haptic feedbacks, which have been proposed during the simulation, have been determined in an experimental way, with the aim of proposing two different effects. The feedback 01 (Max torque $400 \mathrm{mNm}$ ) has been created with the objective of having a free-flowing rotation of the knob; the feedback 02, instead, was characterized by a more jerkily rotation (Max torque $600 \mathrm{mNm}$ ). Fig. 6 shows the two displacement-torque curves relative to one step of the knob.

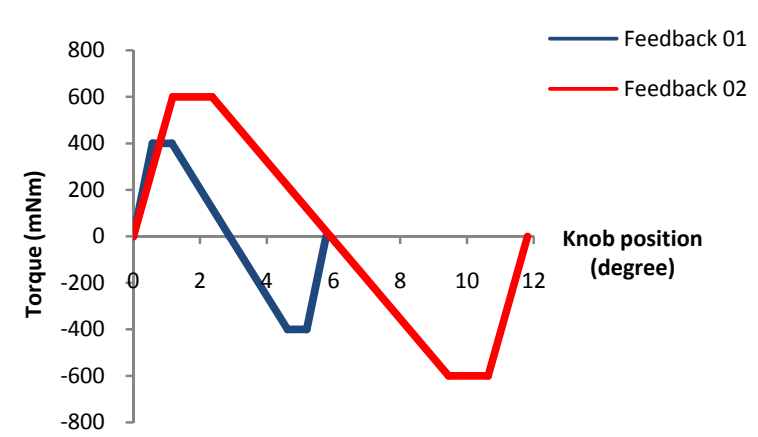

Fig. 6. The dispacement-torque curve of the two haptic feeedbacks.

The minimum and maximum rotation angles have been reasonably settled down as for a temperature control device. The constraints have been respectively $50^{\circ}$ and $310^{\circ}$ in both cases. It is always important to make targeted comparison between two situations and possibly it would be useful to propose one variable per time. In this case, the coefficient of stiffness is maintained steady and the only element that changes is the number of knob steps.

In the most free-flowing case, the number of the settled steps is very high (45). The effect is thus equivalent to an overall stiffness of the rotation, minimizing the perception of the steps. In the second case, we have settled 22 steps, obtaining in this way a different effect. Unfortunately, due to the high inertia provoked by the weight of the knob prototype, a strong value of stiffness originated an anomalous behavior of the knob, and hence, we have preferred to lower the stiffness of the feedback in order to make it more similar to the simulated function.

\subsection{Test protocol}

The test protocol requires that the user had to be provided with some general instructions on the simulation modalities, while he is invited to take place in the driver's seat and to adjust the seating, as it normally happens when sitting in a car. Subsequently, we have requested the fulfillment of some simple tasks, which imply the interaction with the air-conditioning interfaces. The activities carried out during the testing session are summarized below:

- The robotic arm picks up the dashboard prototype and carries it alternatively in the two positions;

- The user touches the dashboard prototype and evaluates the two positions;

- The robotic arm picks the haptic knob and carries it alternatively in the two positions;

- The user rotates the knob and evaluates the two different haptic behaviors in the two positions alternatively.

At first, it has been required to evaluate the comfort of the vehicle in general and of each part of the vehicle, such as seating, steering wheel and pedals. Subsequently, the first layout of the dashboard, named layout 01, has been proposed. Before touching the dashboard prototype and starting the physical interaction with it, the user had to express his impressions related to its placement by only seeing the virtual representation of it. Then, the user had been invited to explore the surface of the dashboard prototype and reaches all its knobs in order to express his impressions related to its placement. The purpose of this procedure was to understand if the perception of the distance is heavily influenced by the Head Mounted Display and by the type of simulation.

The same questions have been then submitted once again for a new configuration of the dashboard prototype, named layout 02, with a diverse placement. Finally, after having quickly reused the first configuration proposed, the user has been asked to state which of the two configurations he prefers.

The second part of the test concerned the use of the haptic knob. The questions focused again on the functioning of the device, which lies in the position corresponding to one of the two cases previously presented to the user. Subsequently, two different feedbacks have been proposed, and the user had to express the pleasantness, effectiveness, and possible changes to be made and in which directions. At the end of the test, the user has been asked to state a clear preference for one of the two proposed feedback modalities.

The users' sample, which has been selected, is made up of 8 male people aged between 20 and 30 with similar morphology and skill on interacting with VR environment in order to not influencing the results of the testing session. The sample is numerically small, but it has been considered sufficient for identifying usability aspects and issues related to our developed system, as described in [26]. Each user had to answer to 30 
questions in total. The time required to carry out all the activities, described before, has been about 20 minutes while the user had to wear the HMD only for 10 minutes, in order to avoid any possible discomfort such as nausea, typically reported in studies on the use of HMDs [28].

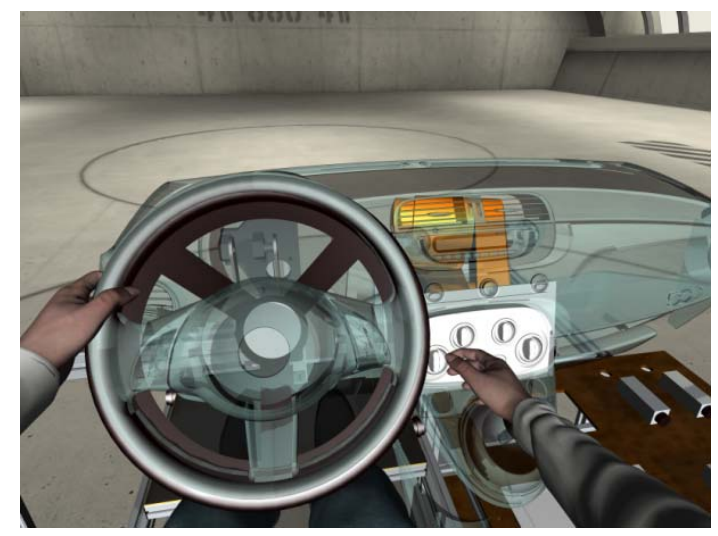

Fig. 7. Rendering of MR environment seen by the urser's point of view.

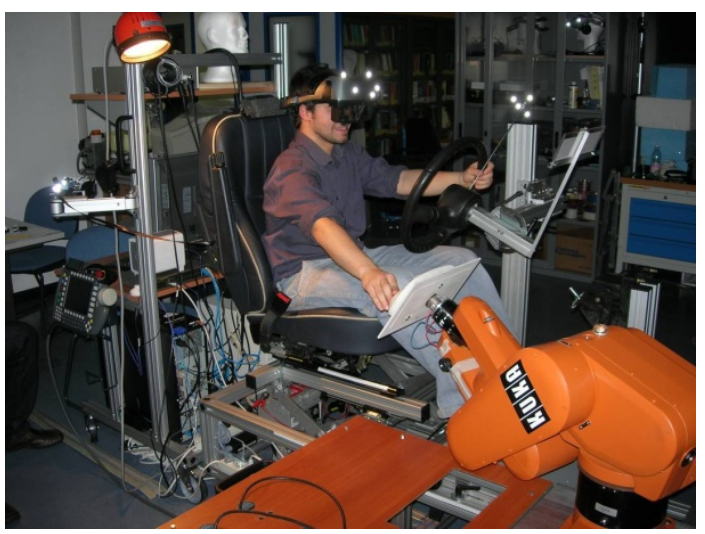

Fig. 8. A user during the execution of a task.

At the end of the tests, some further targeted and explicit questions have been posed, in order to inquire about the general evaluations of the experience. Fig. 7 shows the user's point of view during the evaluation test. Obviously, this figure is a rendering of what the user sees since it is not possible to take a picture from the OST-HMD that shows real and virtual objects. Fig. 8, instead, shows the user during the execution of a task.

\section{DISCUSSION}

In this section we show and discuss the data collected during the testing sessions. In particular, the data refer to the ergonomic evaluations of the two proposed layouts and to the usability aspects of the seating buck system investigated.

Table 4 shows some of the data, presented for a first comparison, related to the first part of the tests, which focused on the evaluation of the general comfort and on the proposed placement of the interfaces.

For a clearer comparison, the evaluations have been divided into two parts. The first part concerns on the mean and the standard deviation of the numerical users' answers. The other part relates to the frequency of the multiple choices answers. From the analysis of the data showed in the table it is possible to deduce some interesting, general considerations.

The interface of the layout 01, in its general position, has been perceived with quite the right height, sometimes in a lower position and slightly far in respect to the actual user position. Even the users, who have judged this distance correct by just observing it, then have stated that it was excessive, after having tried it physically. The configuration of layout 02 has been generally considered as a right position even if some users have defined it as too close.

\section{TABLE 4: RESULTS OF THE TESTING SESSION.}

\begin{tabular}{|l|c|c|}
\hline Assessed aspect & mean & $\boldsymbol{\sigma}$ \\
\hline Steering wheel comfort & 6.75 & 1.30 \\
\hline Pedals comfort & 7.88 & 0.60 \\
\hline General comfort & 7.25 & 0.66 \\
\hline Commands pleasantness & 6.13 & 0.33 \\
\hline Dashboard comfort & 7.38 & 1.32 \\
\hline Position Layout 01 & 7.13 & 1.05 \\
\hline Position Layout 02 & 7.25 & 0.66 \\
\hline
\end{tabular}

Another important aspect, which would be interesting to analyze, is related to the preferences expressed for the two configurations: it has been always considered as the best solution the first one proposed, except for only one case, where the option preferred has been the second one (layout 02). In another single case no one of the two options has been appreciated. Therefore, it seems to be important to investigate into this aspect, trying to inquire in a more rigorous way about to which extent and how the order of the configurations presented to the users affects the evaluations. Actually, we have noticed a strong connection between the order and the preferred configuration. More in general, in 5 cases the configuration of layout 02 has been chosen as the preferred one, in two cases we have a preference for the configuration of the layout 01. By observing these results, it is possible to state that the variation of the original has been generally considered better than the original itself. As regards the two cases in which the layout 01 has been preferred to the layout 02 , it would be necessary to detect if it has occurred the affecting due to the order of the presentation of the two models, or if the preference has been motivated by other factors. We suggest conducting such tests in randomly to eliminate the effects of the presentation order.

Table 5 concerns the comparison of the evaluations of the two different feedbacks proposed for the knob of the temperature control, by using the haptic device.

In order to evaluate in a rigorous manner these answers, it would be necessary to know the type of feedback of the knobs present in the users' cars. On the basis of the results of these tests, we can actually state that the feedback to which we are used strongly affects the evaluation of other kinds of feedbacks.

In general, feedback 01 would have been characterized by a higher stiffness, while the feedback 02 has been sometimes appreciated, but in other cases would have been a little bit stiffer. It is curious to observe that in most of the cases the 
second feedback proposed has been judged more pleasant than the first one proposed. This trend is, hence, in opposition to the trend of the evaluation of the whole interfaces, when the first solution proposed was often preferred to the second one. We have to say that in the first part of the tests, after having showed the second placement, the first one was proposed once again, in order to enable the user to experience again the sensations. In the second part, related to the haptic knob evaluations, the procedure was more rapid and the feeling produced by the sensitivity easier to compare and thus the testers have tried only one time both the solutions. An interesting aspect to analyze would be, again, the relationship between the orders in which the solutions have been presented.

\section{TABLE 5: HAPTIC KNOB FEEDBACK EVALUATION.}

\begin{tabular}{|l|c|c|}
\hline Assessed aspect & mean & б \\
\hline Pleasantness feedback 01 & 3.88 & 0.93 \\
\hline Effectiveness feedback 01 & 5.00 & 0.87 \\
\hline Comfort feedback 01 & 6.50 & 1.50 \\
\hline Pleasantness feedback 02 & 6.13 & 1.05 \\
\hline Effectiveness feedback 02 & 7.00 & 1.00 \\
\hline Comfort feedback 02 & 5.25 & 1.71 \\
\hline
\end{tabular}

It could be added that probably the difference between the question "which is the most pleasant solution" and "which is the most effective one" has been often not understood. Also in this case it will be important to pay more attention to the way in which the questions are posed, introducing a clearer terminology and avoiding questions, which could be ambiguous or not clear. In the table, we have reported the times that one of the two feedbacks has been chosen as more pleasant or effective. In general, we can state that the feedback 02 has been defined as the most pleasant and effective. To improve the haptic feedback, the users have had the possibility of choosing the answer among five options and "slightly stiffer" has been the most selected suggestion.

Table 6 presents the correlation between quantitative data and qualitative usability aspects. The values have been starting from data collected during the testing sessions and to the users' judgments. The results have been expressed on a scale from 1(bad) to 10 (good).

Initially, we have correlated the system learnability to the times that the user had no problems to understand the technical terms used in the questions during the test. This datum has been elaborated starting from the ratio between the number of the answers, given without the need to reformulate the question, and the total number of questions, which are 30 for each testing session. Actually, this datum mainly concerns the adopted protocol but at the same time provided us with useful information related to the terminology to use in describing the system. The result highlights that the terms used are clear and properly describe the system. Obviously, this datum will be more significant when the sample of users will be wider and more heterogeneous since, in this testing session, the involved users are skilled on VR technologies.

The margin of error is an aspect that we have correlated with the errors occurred during the execution of the testing session.
We divided such data in two categories: system and user's errors. The first one represents the times that one of the components constituting the system (robotic arm, tracking system, haptic device, etc.) goes in failure mode during the system configuration. In the second category similar kind of errors are collected but only if they occurred when the user interacts with the system. These values have been elaborated starting from the ratio between number of errors and the number of steps in which we subdivided the testing session, which are 10 for each testing session. We considered the margin of error one of the aspects that can represent the reliability of our system.

TABLE 6: RESULTS OF HEURISTIC EVALUATION.

\begin{tabular}{|c|c|c|c|}
\hline Assessed aspect & mean & $\sigma$ & Usability aspect \\
\hline Correct comprehension & 9,63 & 0,48 & Learnability \\
\hline System errors & 9,75 & 0,43 & \multirow{2}{*}{ Margin of error } \\
\hline User’s errors & 9,13 & 0,60 & \\
\hline Surrounding environment & 8,13 & 0,78 & \multirow{6}{*}{ Efficiency } \\
\hline Seating buck structure & 7,25 & 0,66 & \\
\hline OST-HMD limitation & 3,25 & 1,30 & \\
\hline OST-HMD weight & 2,13 & 0,60 & \\
\hline Field of view limitation & 4,25 & 1,39 & \\
\hline Time for layout configuration & 9,38 & 0,48 & \\
\hline Perceived Comfort & 8,50 & 1,22 & \multirow{3}{*}{ Satisfaction } \\
\hline Perceived Realism & 4,38 & 1,87 & \\
\hline Global evaluation & 7,13 & 1,27 & \\
\hline
\end{tabular}

The efficiency of the system has been correlated to some issues, which can limit the interaction and consequently invalidate the assessment. The surrounding environment and the presence of the seating buck structure, for instance, can influence negatively the user during the testing session since they are visual noises. For this reason, the adopted illumination is important and the choice of using a direct light only to the hand of the user limits such trouble and the average results are very positive. Another issue relates to the efficiency of the system, and in particular for what concerns the use of the OST-HMD. Unfortunately, this device is quite cumbersome and it limits the field of view and movements of the user. Another limitation is due to the OST technology that does not allow a perfect merging between real and virtual objects. Using another kind of MR visualization technology, as the Video See Trough, can improve the merge of real and virtual environments and, consequently, the perceived realism of the user. The time for configuring the seating buck is another issue investigated for assessing the efficiency and the effectiveness of our approach. Thanks to the robotic arm, the time needed for the layout changing is very short (few seconds) and thereby the users are able to correctly compare different proposed solutions and the time need to complete all the testing session (about 20 minutes) did not wearied any user.

In general, the users' judgments have been positive also in relation to the aspects related to the satisfaction in using the system. These results are encouraging and show the effectiveness of our system that certainly improves the normal activities carried out for evaluating car interiors. 


\section{CONCLUSION AND FUTURE WORK}

In this paper we have presented the design and the implementation of a MR seating buck system, which allows simulating different driver's seats to perform some evaluation tests useful for car interior development. Our flexible system allows us to test two different layouts of the car dashboard and compare them in term of most ergonomic position and the best haptic knob behavior. The results of the tests reveal that the users can appreciate the differences of the proposed solutions and that it is easy to identify the best dashboard layout among those proposed.

One of the most important achievements is the prompt configurability of the system, which has been obtained thanks to the use of the robotic arm. All the users have performed the entire test in few minutes, without any particular problems. Some issues related to the MR technologies have to be still solved. In particular, the use of the OST-HMD has been proved poorly performing. The evaluation protocol, which we have elaborated, can be considered suitable for this kind of design assessment. The use of three different types of answers (affirmative/negative, multiple choices, qualitative evaluation) has to be reviewed since sometimes has been difficult to correlate them. The data collected during the tests have also enabled us to assess the usability of the system.

In the next future we aim at improving the automation of the seating buck in order to set the dashboard configuration directly from digital model. We also plan to integrate other dashboard components with the objective of extending the application fields of our system. Finally, we aim at refining our testing protocol, such as Presence Questionnaire (PQ) or similar [29], and extend the users' sample with the purpose of obtaining more reliable results.

\section{ACKNOWLEDGEMENT}

The research work presented in this paper has been supported by the Italian Ministry of University and Research in the frame of the FIRB-PROGIMM project (www.kaemart.it/progimm).

\section{REFERENCES}

[1] R. E. Su, H. J. Xue, B. F. Song. Ergonomic assessment method for cockpit layout of civil aircraft $x$ based on virtual design, in Proc. of 26th Congress of International Council of the Aeronautical Sciences, Anchorage, Alaska, USA, 2008.

[2] W. Lijing, X. Wei, H. Xueli, S. Xiaohui, Y. Jinhai, Z. Lin and S Gaoyong. The virtual evaluation of the Ergonomics layout in Aircraft cockpit, in Proc. of IEEE 10th Int. Conf. Computer-Aided Industrial Design \& Conceptual Design CAID \& CD, pp. 1438-1442, 2009.

[3] H. Zhang and D. Zhuang and F. Wu. The study on pleasure and ergonomics of cockpit interface design, in Proc. of IEEE 10th Int. Conf. Computer-Aided Industrial Design \& Conceptual Design CAID \&CD, pp. 1400-1402, 2009.

[4] C.F. Kuo, and M.J. J.Wang, Interactive virtual evaluation for interface design, Human Factors Manual, vol. 17 pp. 485-495, 2007.

[5] R. Verhoeven and A.de Reus, Prototyping interactive cockpit applications, in Proc. 23rd Digital Avionics Systems Conference, vol.2 , 2004.
[6] L. Woon-Sung, K. Jung-Ha, and C. Jun-Hee, A driving simulator as a virtual reality tool, in Proc. of International Conference on Robotics and Automation, pp. 71-76, 1998.

[7] F. Caputo, G. D. Gironimo, G. Monacelli, and F. Sessa, The design of a virtual environment for ergonomic studies, in Proc. of ADM International Conference, Rimini, 2001.

[8] H. Salzmann and B. Froehlich, The Two-User Seating Buck: Enabling Face-to-Face Discussions of Novel Car Interface Concepts, in Proc. of IEEE Virtual Reality Conference, pp. 75-82, 2008.

[9] M. Moehring, A. Gloystein, and R. Doerner, Issues with Virtual Space Perception within Reaching Distance: Mitigating Adverse Effects on Applications Using HMDs in the Automotive Industry, in Proc. of IEEE Virtual Reality Conference, pp. 223-226, 2009.

[10] O. Toshikazu, K. Tsuyoshi, Y. Hiroyuki, and T. Hideyuki, A Mixed Reality System with Visual and Tangible Interaction Capability Application to Evaluating Automobile Interior Design, in Proc. of 2nd IEEE/ACM International Symposium on Mixed and Augmented Reality, 2003.

[11] M. Bordegoni, G. Caruso, F. Ferrise, and U. Giraudo, A mixed environment for ergonomic tests: tuning of the stereo viewing parameters, in Proc. of Eurographics Italian Chapter Conference, pp. 127-134, 2007.

[12] M. Bordegoni, G. Caruso, and F. Ferrise, Mixed-Reality Environment based on Haptic Control System for a tractor cabin design review, in Proc. of CIRP Design Conference, Enschede, 2008.

[13] SAE, Motor Vehicle Dimensions, Document Number: J1100, September 2005.

[14] M. Astier and N. Mitchell, Package Drawing Exchanges, Global Cars Manufacturers Information Exchange Group, 2008.

[15] www.boschrexroth.com.

[16] R. V. Dukkipati, Mechanism and Machine Theory, 2007.

[17] www.nvisinc.com

[18] www.vicon.com

[19] www.kuka-robotics.com

[20] G. Colombo, F. De Angelis, and L. Formentini, A mixed virtual reality haptic approach to carry out ergonomics tests on virtual prototypes, in Proc. of TMCE, Ljubljana, Slovenia, 2006.

[21] www.3ds.com/products/3dvia/3dvia-virtools

[22] N.S. Kirk and S. Ridgway, Ergonomics testing of consumer products 1. General considerations, Applied Ergonomics, vol. 1, no. 5,pp. 295 - 300, 1970.

[23] J. Nielsen and R. L. Mack, Usability Inspection Methods, published by John Wiley \& Sons, New York, NY, 1994.

[24] J. Nielsen, Evaluating the thinking aloud technique for use by computer scientists, in Hartson, H. R., and Hix, D. (Eds.), Advances in Human-Computer Interaction, Ablex, Norwood, NJ, vol. 3, pp. 69-82, 1992.

[25] J. Nielsen, Guerrilla HCI: using discount usability engineering to penetrate the intimidation barrier, in Cost-justifying usability, Academic Press, Inc., pp. 245-272, 1994.

[26] J. Nielsen, Usability Engineering, published by Morgan Kaufmann, San Francisco, 1993.

[27] www.sae.org

[28] J.P. Rolland, F.A. Biocca, T. Barlow and A. Kancherla, Quantification of adaptation to virtual-eye location in see-thru head-mounted displays, in Proc. of the Virtual Reality Annual International Symposium, 1995.

[29] M. Schuemie, P. van der Straaten, M. Krijn, and C. van der Mast, Research on Presence in Virtual Reality: A Survey, Cyberpsychology and Behavior, vol. 4, pp. 183-201, 2001.

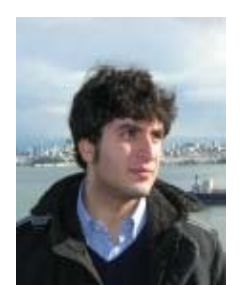

Giandomenico Caruso received a Degree in Mechanical Engineering at the Università della Calabria in Rende (CS), Italy and the Ph.D degree in Virtual Prototyping and Real Products at Politecnico di Milano, Italy, with a thesis entitled: "Industrial product development using Mixed Prototyping". Since 2008 he is Assistant Professor at the Department of Mechanical Engineering of Politecnico di Milano. His research activity focuses on the development and the validation of emerging technologies, related to Virtual and Augmented Reality, to support the development of industrial products. He has participated and participates in several national and European research projects, and in some of them he has acted as coordinator of the research activities. He is author and/or co-author of several scientific articles, mostly published in journals and conference proceedings with international relevance. 